\title{
Viral loads of Delta-variant SARS-CoV-2 breakthrough infections after vaccination and booster with BNT162b2
}

\author{
Matan Levine-Tiefenbrun ${ }^{1,2}$, Idan Yelin ${ }^{1}{ }^{1}$, Hillel Alapi ${ }^{3}$, Rachel Katz ${ }^{3}$, Esma Herzel ${ }^{3}$, Jacob Kuint ${ }^{2,3}$, \\ Gabriel Chodick $\mathbb{D}^{2,3}$, Sivan Gazit $\mathbb{B}^{3}$, Tal Patalon $\mathbb{B}^{3 凶}$ and Roy Kishony $\mathbb{B}^{1,4}{ }^{\infty}$
}

\begin{abstract}
The effectiveness of the coronavirus disease 2019 (COVID-19) BNT162b2 vaccine in preventing disease and reducing viral loads of breakthrough infections (BTIs) has been decreasing, concomitantly with the rise of the Delta variant of severe acute respiratory syndrome coronavirus 2 (SARS-CoV-2). However, it is unclear whether the observed decreased effectiveness of the vaccine in reducing viral loads is inherent to the Delta variant or is dependent on time from immunization. By analyzing viral loads of over 16,000 infections during the current, Delta-variant-dominated pandemic wave in Israel, we found that BTIs in recently fully vaccinated individuals have lower viral loads than infections in unvaccinated individuals. However, this effect starts to decline 2 months after vaccination and ultimately vanishes 6 months or longer after vaccination. Notably, we found that the effect of BNT162b2 on reducing BTI viral loads is restored after a booster dose. These results suggest that BNT162b2 might decrease the infectiousness of BTIs even with the Delta variant, and that, although this protective effect declines with time, it can be restored, at least temporarily, with a third, booster, vaccine dose.
\end{abstract}

The BNT162b2 vaccine (Pfizer/BioNTech) has shown high effi$\mathrm{cacy}^{1}$ in preventing COVID-19 as well as real-world effectiveness for prevention of infection, symptoms, hospitalization, severe disease and death ${ }^{2,3}$. Moreover, we and others have reported that the vaccine reduces viral load of BTIs, suggesting that it can also reduce infectiousness ${ }^{4,5}$. Indeed, national vaccination campaigns were followed by reduced infection rates, with the effect extending beyond the individual vaccinees to the community level ${ }^{6-8}$, and giving rise to hopes for herd immunity and disease eradication. In Israel, a rapid national vaccination campaign (over 50\% inoculated within 3 months) was followed by near-complete eradication of new COVID cases in the period from April to June 2021 ( ref. $^{9}$ ).

However, despite the comprehensive vaccination campaign and its initial dramatic effect, since mid-June 2021, a new surge has occurred in several highly vaccinated countries, including Israel. This increased infection rate, and concomitant increased rate of BTI, comes amid a global spread of the Delta variant of SARS-CoV-2 (B.1.617.2 $)^{10}$ and a possible waning of the vaccine-induced immune response, raising questions of vaccine effectiveness against the Delta variant over time. Considering the time since vaccination, studies have shown waning efficacy in protection against infection after 6 months ${ }^{11,12}$. Nevertheless, to date, only a slight decline has been observed in preventing severe disease and its consequences ${ }^{13}$.
Considering the viral loads of BTIs, reports vary substantially as to whether, and to what extent, the vaccine is effective in reducing viral loads of BTI with the Delta variant ${ }^{14,15}$.

Given this uncertainty, to restrain the current wave of the pandemic, Israel, followed by the United States and the United Kingdom, has decided to offer and recommend a third dose (or booster) to people for whom 5 months have passed since their second dose. In Israel, the booster dose was first offered to people aged 60 years or older and then gradually extended to younger age groups. First reports of real-world data in humans indicate that the booster dose is effective in preventing infections ${ }^{16}$. Similarly, a booster study in primates against the Beta variant using the mRNA-1273 vaccine has shown up to a 20 -fold increase in neutralizing antibodies ${ }^{17}$. However, it is still unclear how the booster shot affects the viral load in BTI in humans and in Delta-dominant real-world settings.

In this study, we retrospectively collected and analyzed the reverse transcription quantitative polymerase chain reaction (RT-qPCR) test measurements of three SARS-CoV-2 genes- $E, N$ and $R d R p$ (Allplex 2019-nCoV assay, Seegene)-from positive tests of patients at Maccabi Healthcare Services. We focus on infections of adults over age 20 between 28 June and 9 September 2021, when Delta was the dominant variant in Israel (over 93\%) ${ }^{9}$. Crossing this dataset with vaccination data (59\% of Israel's population was two-dose vaccinated by 28 June), we identified, in total, 3,100 infections of unvaccinated, 12,934 BTIs of two-dose-vaccinated and 519 BTIs of booster-vaccinated individuals (Methods: 'Vaccination status'; Table 1).

Considering all of these infections $(n=16,553)$, we built a multivariable linear regression model for the cycle threshold $(\mathrm{Ct})$ value of each of three SARS-CoV-2 genes, accounting for vaccination at different time bins before the infection and for receiving the booster, as well as adjusting for sex, age and calendric date. Focusing on the $R d R p$ gene, regression coefficients for vaccinated over unvaccinated, representing the difference in $\mathrm{Ct}$ between the groups, started with 4.6 (95\% confidence interval (CI), 2.2-6.9) for BTI 7-30 d after the second vaccine dose, yet decayed over time down to 0.6 (95\% CI, 0.05-1.12) after about 2 months $(P=0.00005$, Methods: 'Change in Ct over time'), and vanished to insignificant values for infections 6 months or longer after vaccination. Analyzing data for up to $34 \mathrm{~d}$ after inoculation revealed that this decline, however, was overturned after the booster shot, which was associated with an increase of 2.4 (95\% CI, 2.0-2.9) in the Ct. Noting that a difference of $1 \mathrm{Ct}$ unit is approximately equivalent to a factor of 2 in the

'Faculty of Biology, Technion - Israel Institute of Technology, Haifa, Israel. ${ }^{2}$ Sackler Faculty of Medicine, Tel-Aviv University, Tel-Aviv, Israel. ${ }^{3}$ Maccabitech, Maccabi Health Services, Tel Aviv, Israel. ${ }^{4}$ Faculty of Computer Science, Technion - Israel Institute of Technology, Haifa, Israel. 凶e-mail: patalon_t@mac.org.il; rkishony@technion.ac.il 


\section{Table 1 | Study population}

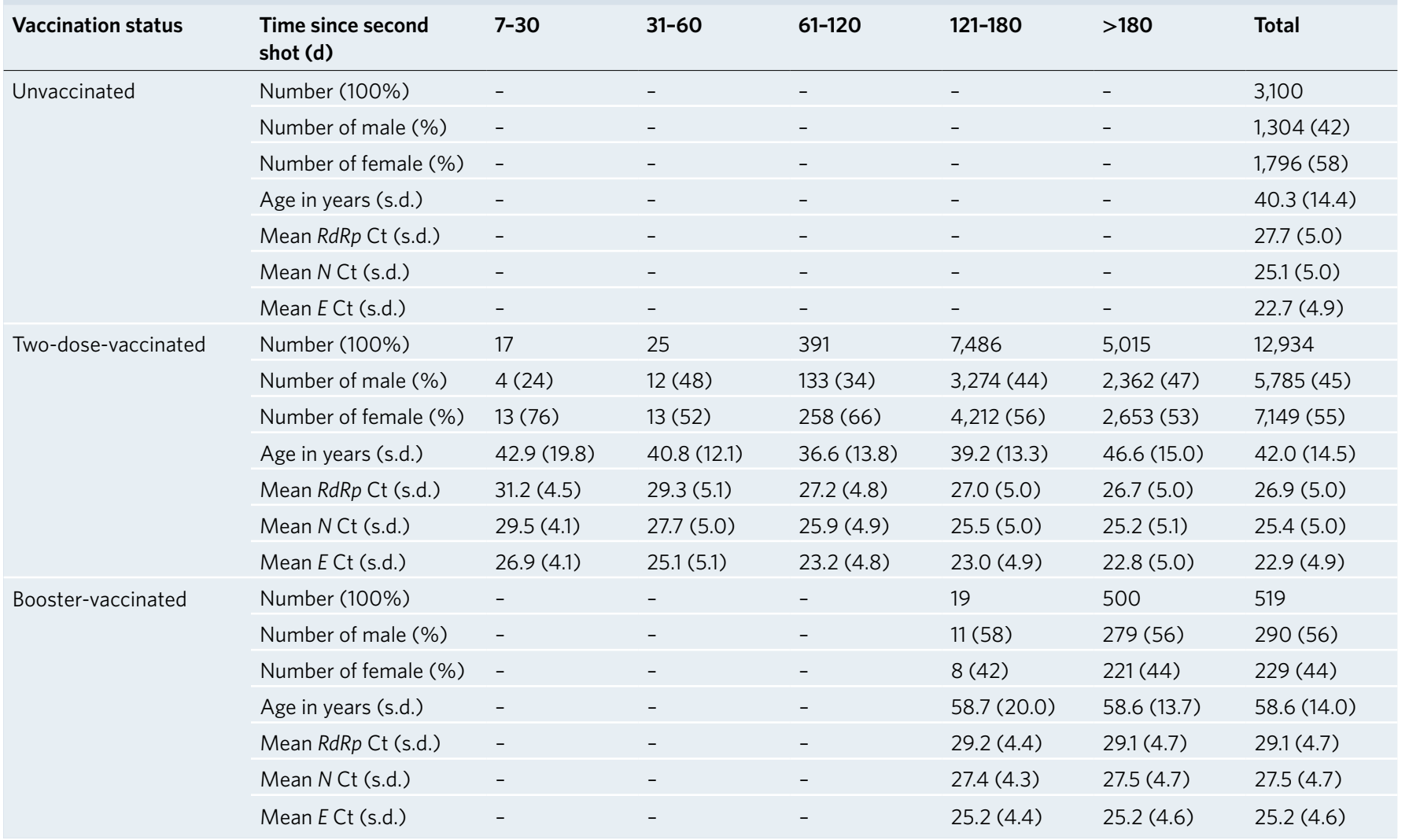

number of viral particles per sample, this increase in the Ct corresponds to a more than five-fold reduction in viral load (Methods: 'Linear regression'; Fig. 1a). Similar changes in Ct were also observed for the two other genes, $N$ and $E$ (Extended Data Fig. 1). Although the model adjusts for sex and age, noting that, due to national rollout guidelines, the average age of the booster group was higher than that of the two-dose-vaccinated group (58.6 versus 42.0 years old), we also repeated the multivariable linear regression analysis separately for patients older and younger than age 50 , which yielded similar results (Extended Data Fig. 2). Furthermore, as full protection might not always be fully realized $7 \mathrm{~d}$ after either the second dose or booster dose ${ }^{16}$, we repeated the analysis considering time bins starting $14 \mathrm{~d}$ after these doses and observed similar associations (Extended Data Fig. 3). To avoid possible effects of severe cases on the results, we also analyzed the data while excluding hospitalized patients, obtaining similar results (Extended Data Fig. 4). Finally, we note that, because during the current surge only a small fraction of patients is within their initial 2-month post-vaccination period, considering the entire population as a whole, there is only a very small viral load difference between the vaccinated and unvaccinated groups (0.22 (95\% CI, 0.02-0.42); Fig. 1b).

Our results show that the vaccine is initially effective in reducing viral loads of Delta BTIs, with a magnitude of ten-fold (95\% CI, 4-30) (average over the first 2 months after vaccination), consistent with its initial effectiveness against pre-Delta variants ${ }^{4,5}$. However, this viral load reduction effectiveness declines with time after vaccination, significantly decreasing at 3 months after vaccination and effectively vanishing after about 6 months. As the Delta variant surfaced when a large fraction of the vaccinated population was already past the initial 2-month post-vaccination period, the population-wide average effect of the vaccine on Delta viral loads is negligible, consistent with and explaining reports ${ }^{14,15}$ of no difference in $\mathrm{Ct}$ between vaccinated and unvaccinated individuals infected with Delta. Consistently, we found that a booster shot is associated with a regained viral load reduction even during a Delta-dominated surge, suggesting restored vaccine-associated mitigation of transmissibility. In total, these results suggest that, although the initial vaccine effectiveness in reducing viral loads of Delta-variant BTIs is similar to its observed effectiveness in pre-Delta surges, this effectiveness is reduced with post-vaccination time and can then be restored with a third booster dose, consistent with immunity against infection waning and third inoculation boosting.

Our study had several limitations. First, although viral load is a common proxy for infectiousness, positive PCR results do not necessarily imply a viable virus, and a correlation between viral loads and infectiousness is not fully established. Second, recent studies suggested that a faster decline in viral load occurs in vaccinated compared to unvaccinated individuals with Delta infection ${ }^{14,15}$, offering a potential differentiation effect on $\mathrm{Ct}$ for samples taken long after the onset of symptoms. It is, therefore, also possible that the difference in Ct between vaccinated and unvaccinated individuals reflects not only an initial difference in viral loads between these two groups but also a differential viral load decay between the groups. However, we note that we minimized this effect by considering only the first positive test for each patient, which is typically performed within $5-6 \mathrm{~d}$ after infection, namely $1-2 \mathrm{~d}$ after symptoms ${ }^{16,18,19}$. Third, it is unknown how many of the infections in our dataset were symptomatic or asymptomatic, and the fraction of symptomatic infections in the unvaccinated population could be higher. Lastly, potential socio-behavioral differences between vaccinated and unvaccinated individuals might also affect the composition of our dataset.

It is yet to be seen for how long the booster's renewed effect of reducing BTI viral loads will last and whether there will be a need for additional booster doses in the future against the same variant or others to come. Furthermore, vaccine effectiveness against severe infections, although correlated with viral loads ${ }^{20}$, might wane at 
a

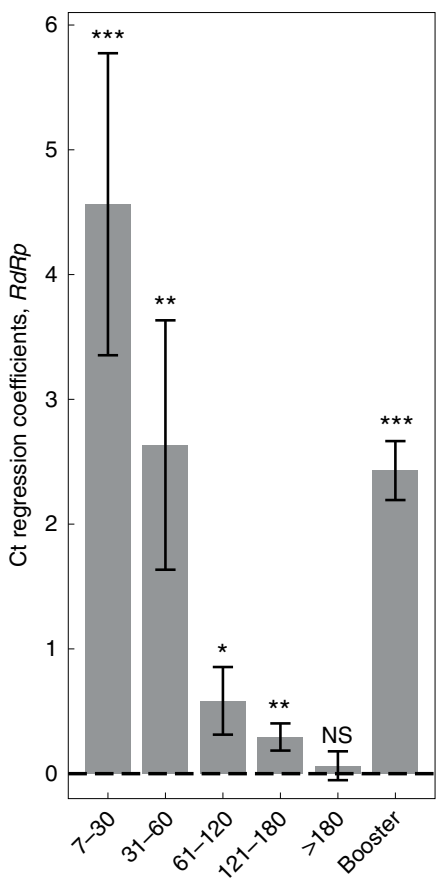

Days since second dose b

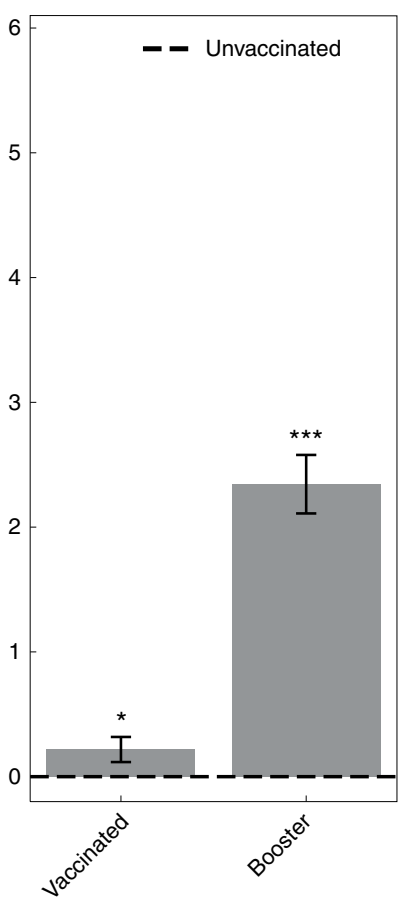

Vaccination status

Fig. 1 | Association of infection $\mathrm{Ct}$ with two-dose vaccination and with the booster. a, Ct regression coefficients, indicating an infection $\mathrm{Ct}$ relative to unvaccinated control group (dashed line), show an initial increase in $\mathrm{Ct}$ in the first 2 months after the second vaccination dose, which then gradually diminishes, ultimately vanishing for infections occurring 6 months or longer after vaccination. Increased $\mathrm{Ct}$ is restored after the booster (right bar). Coefficients were obtained by multivariate linear regression analysis, adjusting for age and sex (Methods; $n=16,553$ ). b. Same model as in $\mathbf{a}$ but without binning post-vaccination times. Because most of the vaccinated population during the current surge are more than 2 months after their second vaccine shot (Table 1), at the whole population level the average effect of the vaccine on $\mathrm{Ct}$ is negligible. Error bars represent one standard error. ${ }^{\star} P<0.05,{ }^{* \star} P<0.01,{ }^{\star \star \star} P<0.001$; all $P$ values are two sided. Data are shown for $C t$ of the RdRp gene; for genes $N$ and $E$, see Extended Data Fig. 1a, b. NS, not significant.

different time scales and be affected differently by booster inoculation, as, to date, only a slight decline has been observed in preventing severe disease $\mathrm{e}^{13}$. Nonetheless, at least in the short term, the lower viral load among booster-vaccinated Delta BTI points to lower infectiousness which, together with other means, such as social distancing and masks, could help impede the spread of the pandemic.

\section{Online content}

Any methods, additional references, Nature Research reporting summaries, source data, extended data, supplementary

information, acknowledgements, peer review information; details of author contributions and competing interests; and statements of data and code availability are available at https://doi.org/10.1038/ s41591-021-01575-4.

Received: 27 August 2021; Accepted: 8 October 2021; Published online: 2 November 2021

\section{References}

1. Polack, F. P. et al. Safety and efficacy of the BNT162b2 mRNA Covid-19 vaccine. N. Engl. J. Med. 383, 2603-2615 (2020).

2. Dagan, N. et al. BNT162b2 mRNA Covid-19 vaccine in a nationwide mass vaccination setting. N. Engl. J. Med. 384, 1412-1423 (2021).

3. Chodick, G. et al. Assessment of effectiveness of 1 dose of BNT162b2 vaccine for SARS-CoV-2 infection 13 to 24 days after immunization. JAMA Netw. Open 4, e2115985 (2021).

4. Levine-Tiefenbrun, M. et al. Initial report of decreased SARS-CoV-2 viral load after inoculation with the BNT162b2 vaccine. Nat. Med. 27, 790-792 (2021)

5. Petter, E. et al. Initial real world evidence for lower viral load of individuals who have been vaccinated by BNT162b2. Preprint at https://www.medrxiv. org/content/10.1101/2021.02.08.21251329v1 (2021).

6. Milman, O. et al. Community-level evidence for SARS-CoV-2 vaccine protection of unvaccinated individuals. Nat. Med. 27, 1367-1369 (2021).

7. Gazit, S. et al. BNT162b2 mRNA vaccine effectiveness given confirmed exposure; analysis of household members of COVID-19 patients. Preprint at https://www.medrxiv.org/content/10.1101/2021.06.29.21259579v1 (2021).

8. Rossman, H. et al. COVID-19 dynamics after a national immunization program in Israel. Nat. Med. 27, 1055-1061 (2021).

9. Our World in Data. https://ourworldindata.org/

10. Lopez Bernal, J. et al. Effectiveness of Covid-19 vaccines against the B.1.617.2 (Delta) variant. N. Engl. J. Med. 385, 585-594 (2021).

11. Mizrahi, B. et al. Correlation of SARS-CoV-2 breakthrough infections to time-from-vaccine; preliminary study. Preprint at https://www.medrxiv.org/co ntent/10.1101/2021.07.29.21261317v1 (2021).

12. Goldberg, Y. et al. Waning immunity of the BNT162b2 vaccine: a nationwide study from Israel. Preprint at https://www.medrxiv.org/content/10.1101/2021. 08.24.21262423v1 (2021).

13. Rosenberg, E. S. New COVID-19 cases and hospitalizations among adults, by vaccination status-New York, May 3-July 25, 2021. MMWR Morb. Mortal. Wkly. Rep. 70, 1306-1311 (2021).

14. Chia, P. Y. et al. Virological and serological kinetics of SARS-CoV-2 Delta variant vaccine-breakthrough infections: a multi-center cohort study. Preprint at https://www.medrxiv.org/content/10.1101/2021.07.28.21261295v1 (2021).

15. Kissler, S. M. et al. Viral dynamics of SARS-CoV-2 variants in vaccinated and unvaccinated individuals. Preprint at https://www.medrxiv.org/content/10.110 1/2021.02.16.21251535v3 (2021)

16. Bar-On, Y. M. et al. Protection of BNT162b2 vaccine booster against Covid-19 in Israel. N. Engl. J. Med. 385, 1393-1400 (2021).

17. Corbett, K. S., Gagne, M., Wagner, D. \& O’Connell, S. Protection against SARS-CoV-2 Beta variant in mRNA-1273 boosted nonhuman primates. Preprint at https://www.biorxiv.org/content/10.1101/2021.08.11. $456015 \mathrm{v} 1$ (2021).

18. Levine-Tiefenbrun, M. et al. Association of COVID-19 RT-qPCR test false-negative rate with patient age, sex and time since diagnosis. Preprint at https://www.medrxiv.org/content/10.1101/2020.10.30.20222935v2 (2020).

19. Xin, H. et al. The incubation period distribution of coronavirus disease 2019 (COVID-19): a systematic review and meta-analysis. Clin. Infect. Dis. https://doi.org/10.1093/cid/ciab501 (2021).

20. Fajnzylber, J. et al. SARS-CoV-2 viral load is associated with increased disease severity and mortality. Nat. Commun. 11, 5493 (2020).

Publisher's note Springer Nature remains neutral with regard to jurisdictional claims in published maps and institutional affiliations.

(C) The Author(s), under exclusive licence to Springer Nature America, Inc. 2021 


\section{Methods}

Data collection. Anonymized SARS-CoV-2 RT-qPCR Ct values were retrieved for all of the positive samples taken between 28 June and 9 September 2021 and tested at the Maccabi Healthcare Services central laboratory using SQL Server Management Studio, version 18.5.1. No statistical method was used to predetermine sample size. Patient data included sex, year of birth, any record of COVID-associated hospitalization and indication of immunosuppression. For patients with multiple positive tests, only the first positive test was included. Vaccination dates for these patients were retrieved from the centralized database of Maccabi Healthcare Services. Immunocompromised patients were excluded $(n=720)$. Patients were excluded if their first positive sample was between the first shot and 1 week after the second shot. Patients with a first positive sample within the first 1 week after the booster shot were also excluded. Patients with positive test results before the study period were also excluded. For each test, Ct values for $E$ gene, $R d R p$ gene, $N$ gene and the internal control were determined using Seegene proprietary software for the Allplex 2019-nCoV assay after the standard oro-nasopharyngeal swab specimen collection procedure. The same PCR model was used for all of the tests (Bio-Rad CFX96 Real-Time PCR Detection System).

Vaccination status. Patients who tested positive $7 \mathrm{~d}$ or more after the second shot were regarded as 'vaccinated. Patients who tested positive $7 \mathrm{~d}$ or more after the third shot were regarded as 'booster-vaccinated'. Patients who tested positive fewer than $7 \mathrm{~d}$ after the second shot were excluded, as were patients who tested positive fewer than $7 \mathrm{~d}$ after their booster shot.

Change in Ct over time. To calculate the change in Ct between post-vaccination time bins, we applied the same linear regression model as above, except replacing the length 5 one-hot vector of post-vaccination time with a length 3 time-accumulated binary vector indicating $[0,0,0],[1,0,0],[1,1,0]$ and $[1,1,1]$ for infections in unvaccinated or 7-60 d, 61-180 d and over $180 \mathrm{~d}$ after the second vaccination. The coefficient of the second binary variable, indicating the difference in Ct between infections in the first 2 months and infections 2-6 months after vaccination is -3.1 ( $95 \% \mathrm{CI},(-4.6)$ to $(-1.6)), P=0.00005$ for the $R d R p$ gene; -2.9 $(95 \% \mathrm{CI},(-4.3)$ to $(-1.4)), P=0.0002$ for the $E$ gene; and -3.0 (95\% CI, $(-4.5)$ to $(-1.4)), P=0.0002$ for the $N$ gene.

Statistics. Linear regression. For each of the three viral genes, we calculated the linear regression of $\mathrm{Ct}$ values as a function of time since the second shot (length 5 one-hot vector indicating 0/1 for time bins of 7-30 d (or 14-30 for Extended Data Fig. 3), 31-60 d, 61-120 d, 121-180 d and more than $180 \mathrm{~d}$ after the second shot; all 0 s for unvaccinated), booster status (0/1), sex (0/1, female/male), age (bins of 10 years) and calendric date (number of days since 28 June 2021). Models were implemented using Python's statsmodels library, version 0.9.0, and scipy library, version 1.1.0.

Ethics committee approval. This study was approved by the Maccabi Healthcare Services institutional review board (IRB). Owing to the retrospective design of the study, informed consent was waived by the IRB, and all identifying details of the participants were removed before computational analysis.

Reporting Summary. Further information on research design is available in the Nature Research Reporting Summary linked to this article.

\section{Data availability}

According to Israel Ministry of Health regulations, individual-level data cannot be shared openly. Specific requests for remote access to de-identified data should be referred to KSM, the Maccabi Healthcare Services Research and Innovation Center Requests sent to Sarit Chehanowitz (chehanow_s@mac.org.il)will be considered within $21 \mathrm{~d}$ pending IRB approval and Maccabi Healthcare Services regulations. Source data are provided with this paper.

\section{Code availability}

The code for the analysis is available at https://github.com/matanlevine/ Viral-loads-of-Delta-breakthrough-and-booster.

\section{Acknowledgements}

This work was supported by the Israel Science Foundation (grant no. 3633/19 to R. Kishony and G.C.) as part of the KillCorona-Curbing Coronavirus Research Program. The funders had no role in study design, data collection and analysis, decision to publish or preparation of the manuscript.

\section{Author contributions}

Study design: M.L.-T., I.Y., T.P., S.G., G.C. and R. Kishony. Data collection: M.L.-T., I.Y., E.H., R. Katz and H.A. Data analysis: M.L.-T., I.Y. and R. Kishony. Data interpretation: M.L.-T., I.Y., J.K., T.P., S.G., G.C. and R. Kishony. Writing: M.L.-T., I.Y. and R. Kishony, with comments from all authors.

\section{Competing interests}

The authors declare no competing interests.

\section{Additional information}

Extended data is available for this paper at https://doi.org/10.1038/s41591-021-01575-4

Supplementary information The online version contains supplementary material available at https://doi.org/10.1038/s41591-021-01575-4.

Correspondence and requests for materials should be addressed to Tal Patalon or Roy Kishony.

Peer review information Nature Medicine thanks the anonymous reviewers for their contribution to the peer review of this work. Alison Farrell is the primary editor on this article and managed its editorial process and peer review in collaboration with the rest of the editorial team.

Reprints and permissions information is available at www.nature.com/reprints. 


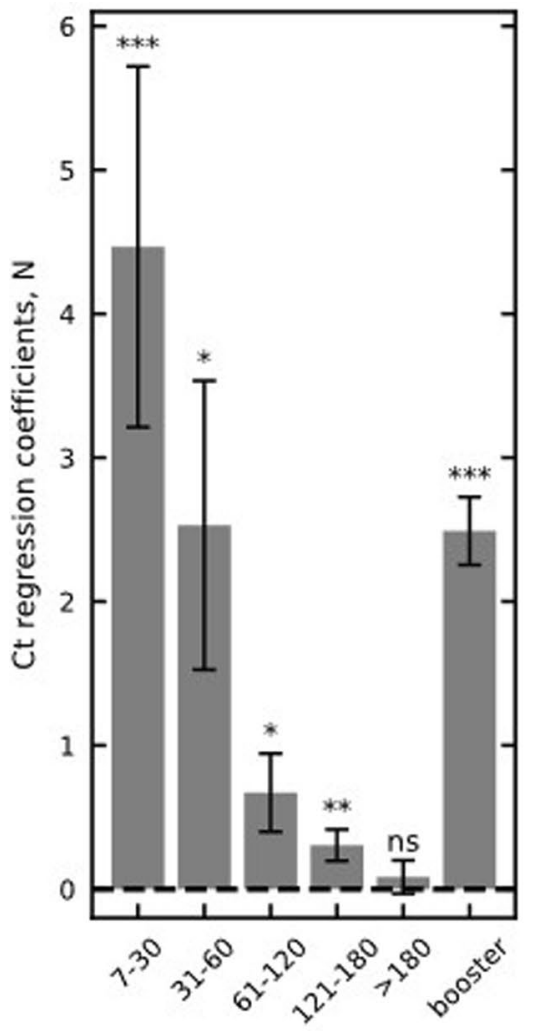

Days since 2 nd dose

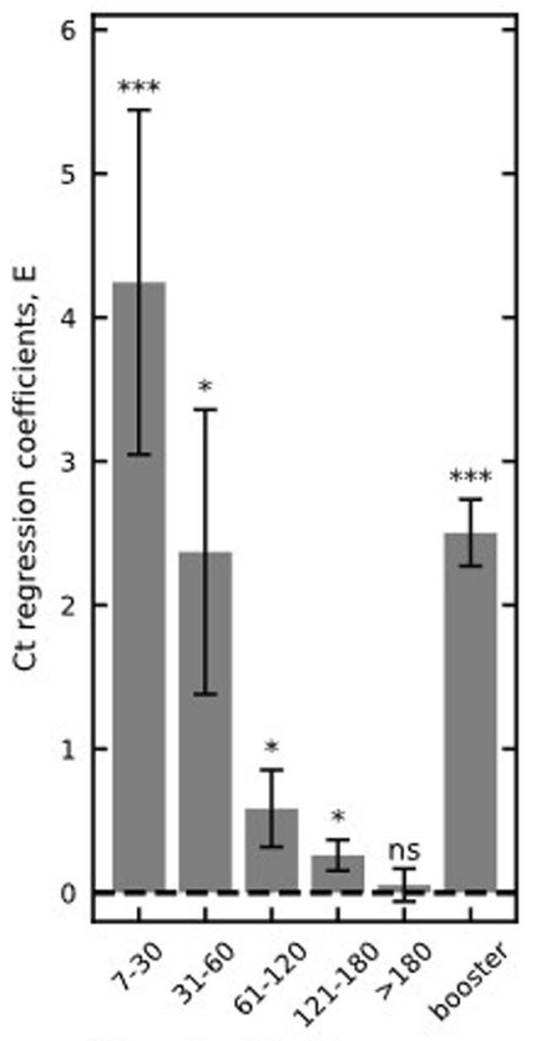

Days since 2 nd dose
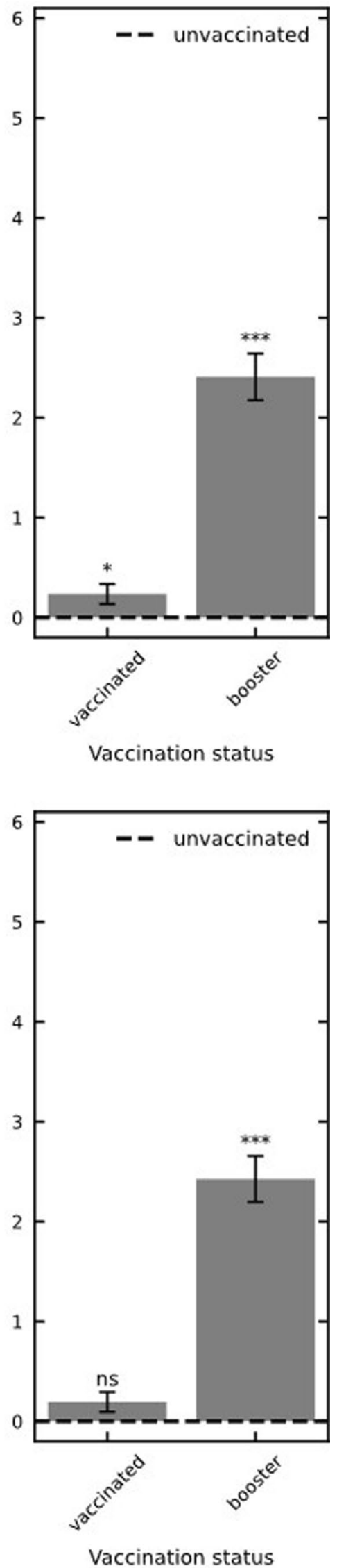

Extended Data Fig. 1 | See next page for caption. 
Extended Data Fig. 1 | Association of infection $\mathbf{C t}$ with 2-dose vaccination and with the booster, for the $\mathbf{N}$ and $\mathbf{E}$ genes. Left, Ct regression coefficients of the $N$ gene (top) and the $E$ gene (bottom), indicating an infection Ct relative to unvaccinated control group (dashed line). Coefficients obtained by multivariate linear regression analysis adjusting for age and sex (Methods; $n=16,553$ ). Right, Same model but without binning post-vaccination times. Error bars represent one standard error. ${ }^{\star}$ - P-value $<0.05,{ }^{\star \star}$ - P-value $<0.01,{ }^{\star \star \star}$ - P-value $<0.001$; all P-values are two-sided. 
a

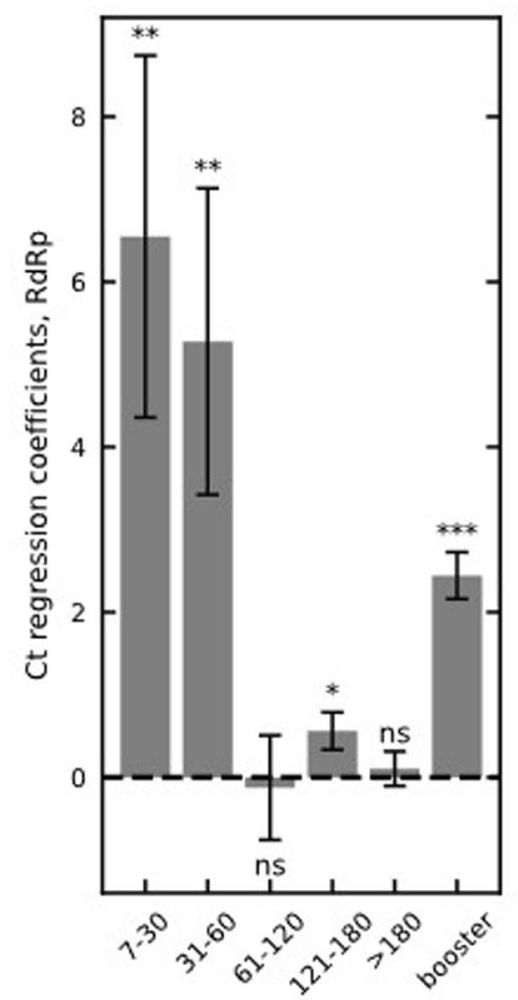

Days since 2 nd dose

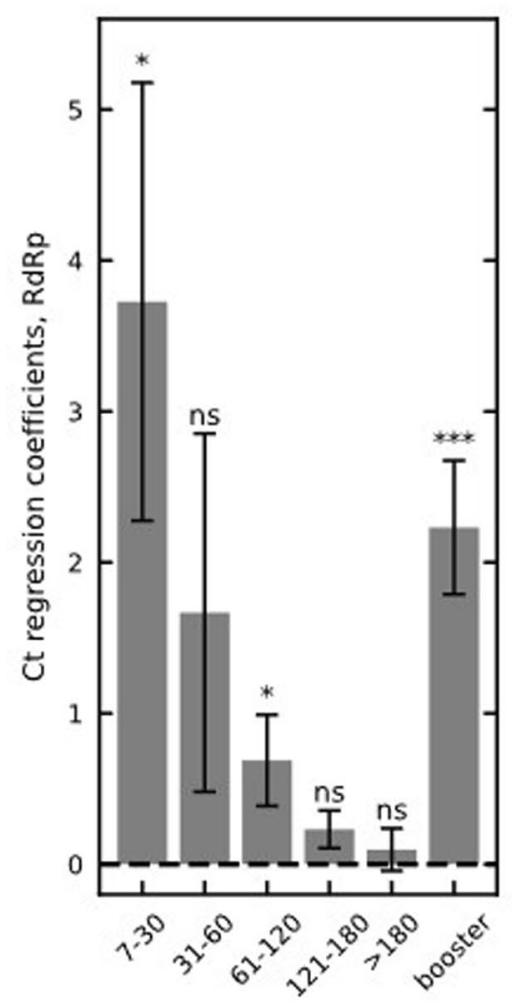

Days since 2 nd dose b

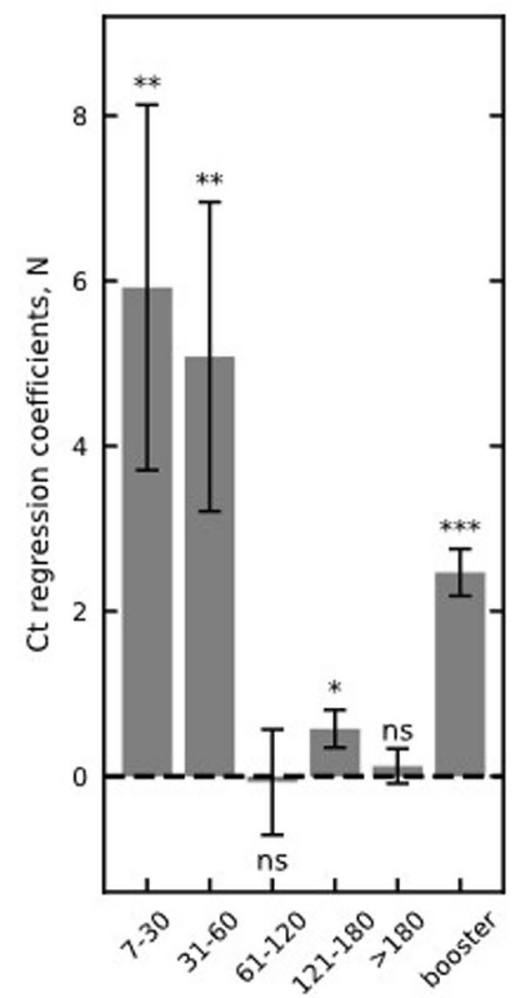

Days since 2 nd dose

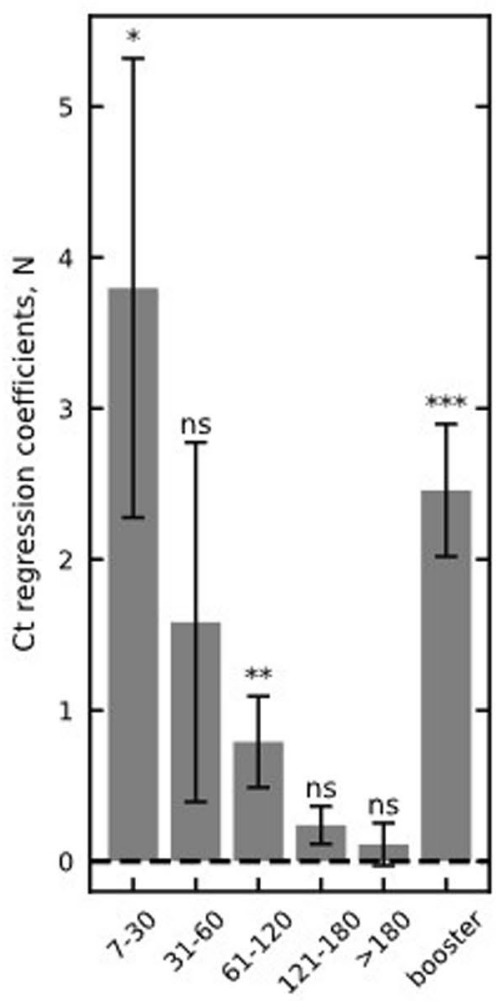

Days since 2 nd dose
C

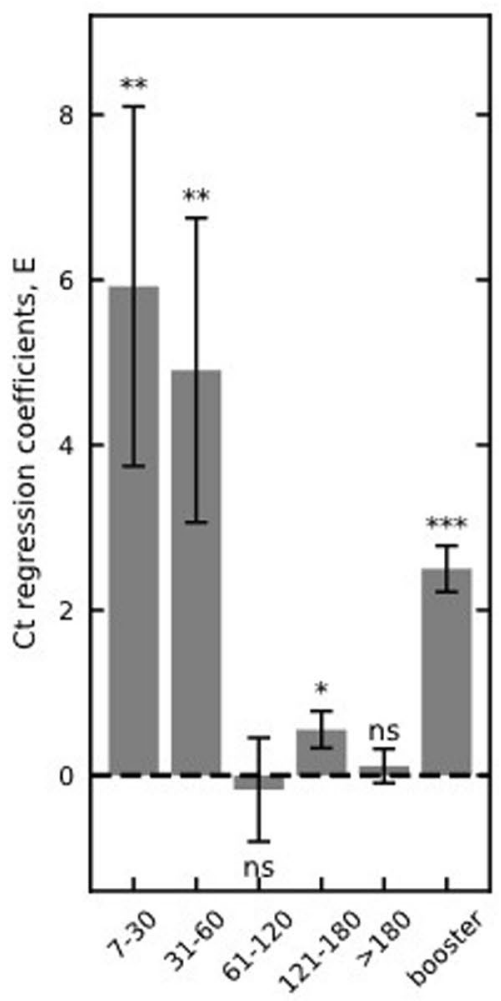

Days since 2nd dose

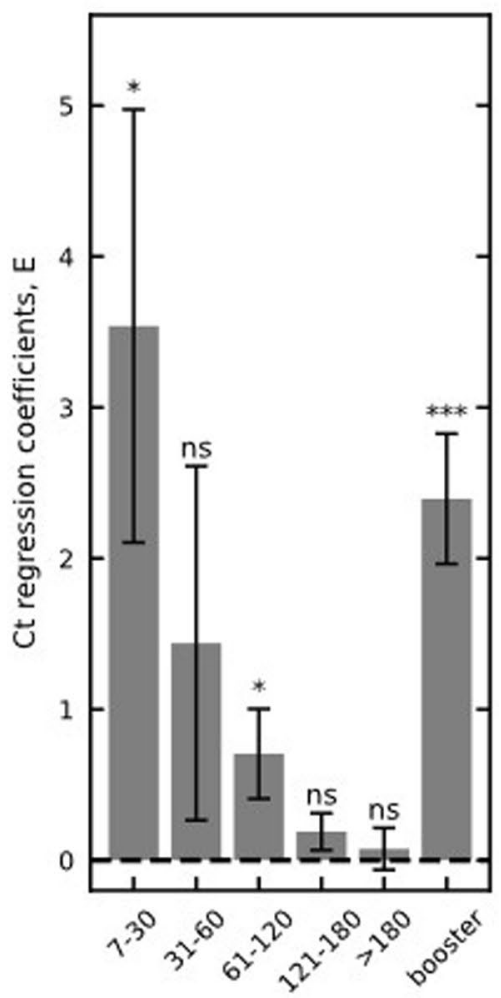

Days since 2nd dose

Extended Data Fig. 2 | See next page for caption. 
Extended Data Fig. 2 | Regression coefficients for the association of Ct with 2-dose vaccination and with the booster for patients older and younger than 50. Same model as in Fig. 1 and Extended Data Figure 1, but restricting for patients 50 years or older (upper panels; $n=4,848$ ) and for patients younger than 50 (lower panels; $n=11,705$ ). Ct regression coefficients are shown for the $R d R p(a), N(b)$ and $E(c)$ genes. Error bars represent one standard error. ${ }^{\star}$ - P-value $<0.05,{ }^{\star \star}$ - P-value $<0.01,{ }^{\star \star \star}$ - P-value $<0.001$; all P-values are two-sided. 
a

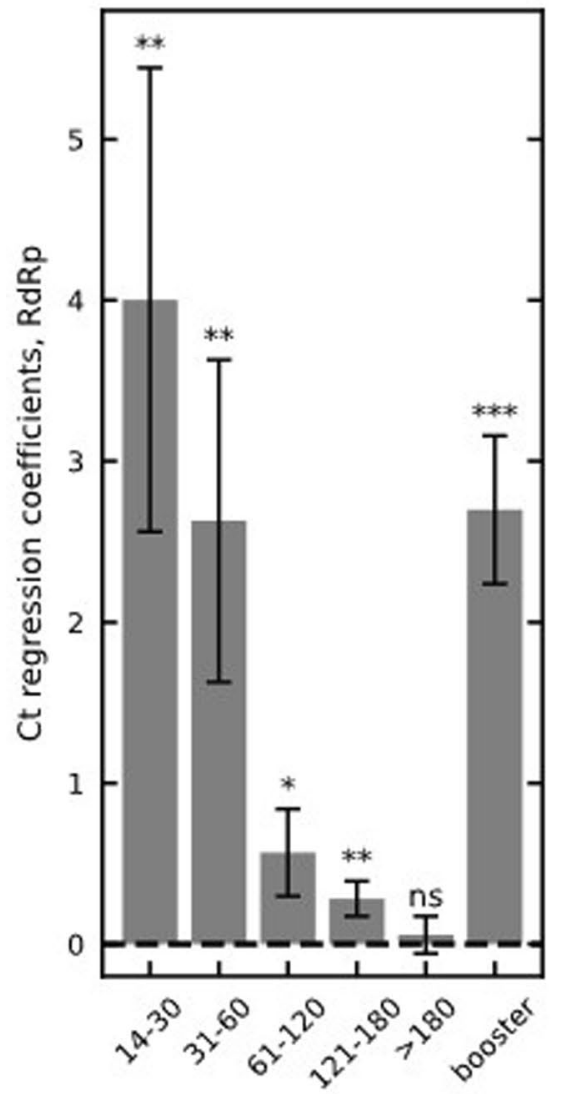

Days since 2 nd dose b

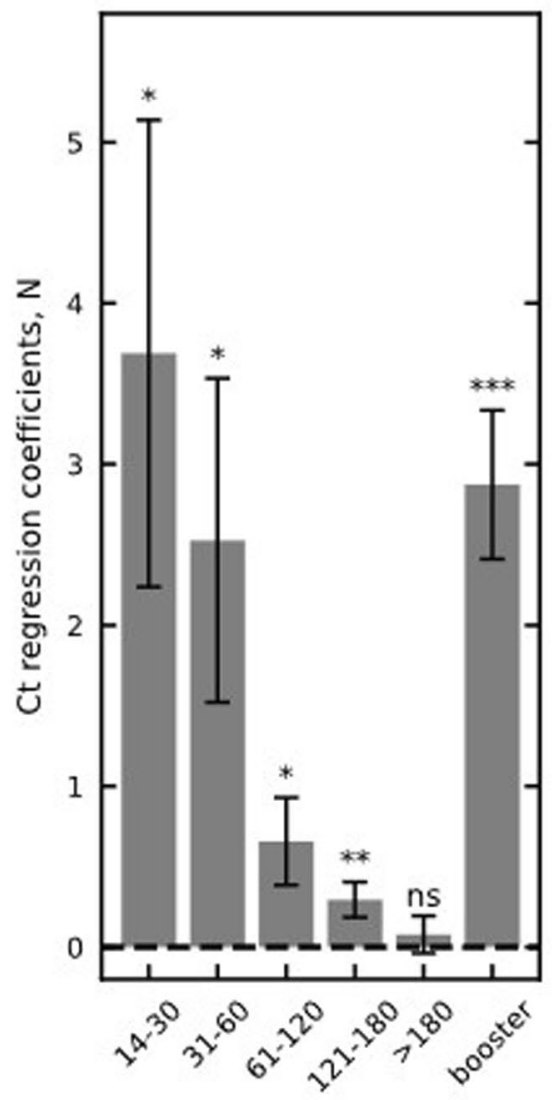

Days since 2nd dose
C

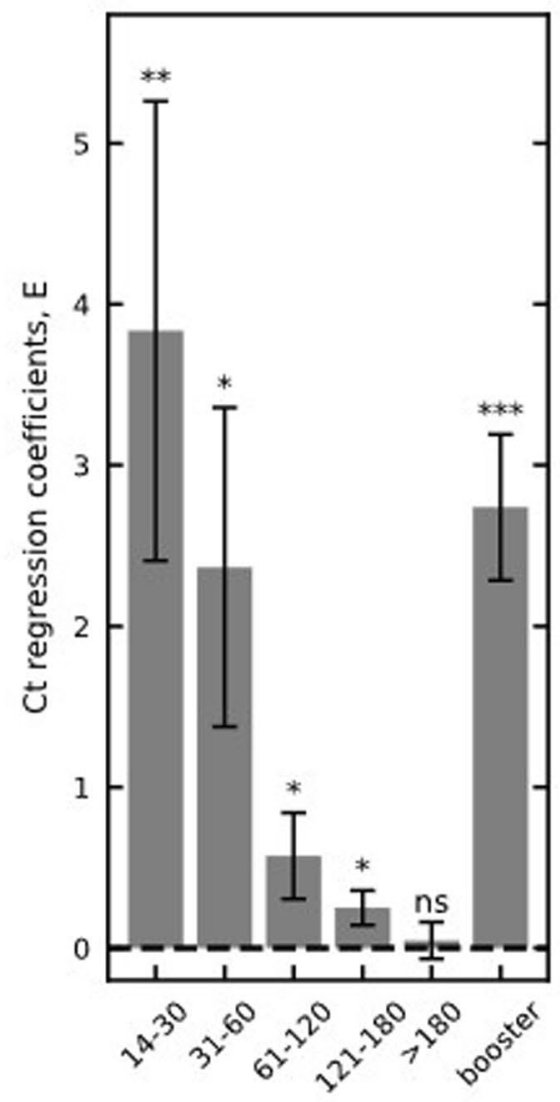

Days since 2 nd dose

Extended Data Fig. 3 | Regression coefficients for the association of Ct with 2-dose vaccination and with the booster starting from 14 days after inoculation. Same model as in Fig. 1 and Extended Data Figure 1, but including positive test results starting from 14 days after inoculation instead of 7 days $(n=16,160)$. Ct regression coefficients are shown for the $R d R p(a), N(b)$ and $E(c)$ genes. Error bars represent one standard error. ${ }^{\star}-P$-value $<0.05$, ${ }^{\star \star}$ - P-value $<0.01,{ }^{\star \star \star}$ - P-value $<0.001$; all P-values are two-sided. 
a

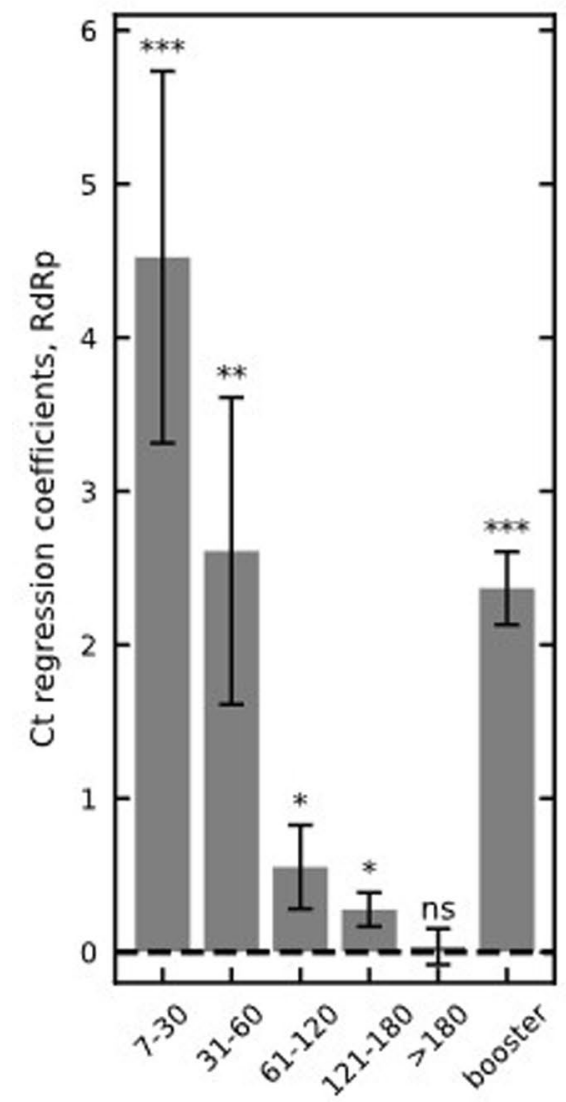

Days since 2 nd dose

b

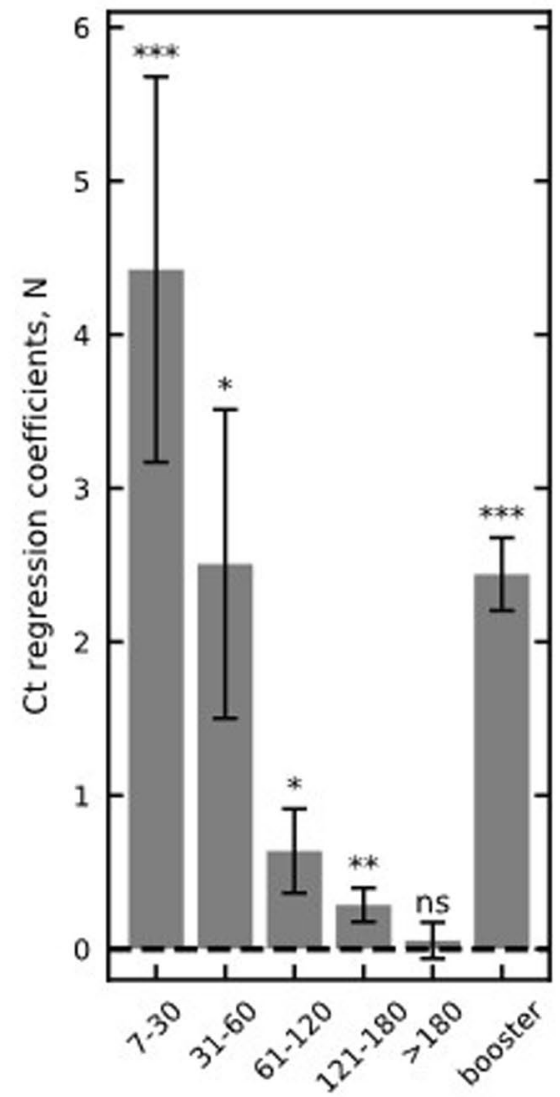

Days since 2 nd dose

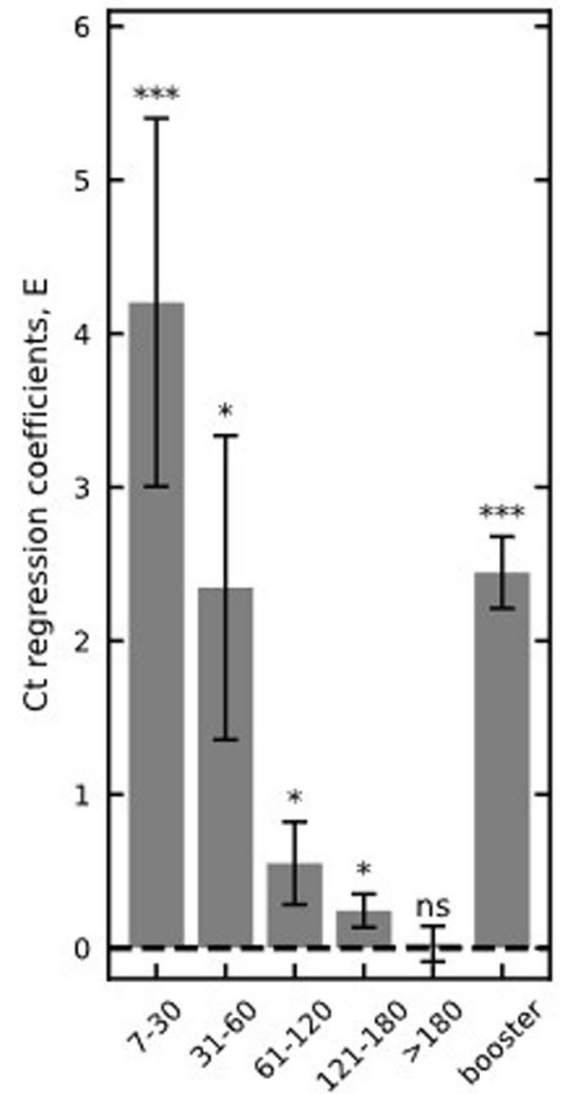

Days since 2 nd dose

Extended Data Fig. 4 | Regression coefficients for the association of Ct with 2-dose vaccination and with the booster for non hospitalized patients. Same model as in Fig. 1 and Extended Data Figure 1, but excluding patients hospitalized due to COVID-19 $(n=16,322)$. Ct regression coefficients are shown for the RdRp (a), N (b) and E (c) genes. Error bars represent one standard error. ${ }^{\star}$ - P-value $<0.05$, ${ }^{\star \star}$ - P-value $<0.01$, ${ }^{\star \star \star}-\mathrm{P}$-value $<0.001$; all P-values are two-sided. 


\section{Reporting Summary}

Nature Portfolio wishes to improve the reproducibility of the work that we publish. This form provides structure for consistency and transparency in reporting. For further information on Nature Portfolio policies, see our Editorial Policies and the Editorial Policy Checklist.

\section{Statistics}

For all statistical analyses, confirm that the following items are present in the figure legend, table legend, main text, or Methods section.

$\mathrm{n} / \mathrm{a} \mid$ Confirmed

$\bigotimes$ The exact sample size $(n)$ for each experimental group/condition, given as a discrete number and unit of measurement

Х $\square$ A statement on whether measurements were taken from distinct samples or whether the same sample was measured repeatedly

The statistical test(s) used AND whether they are one- or two-sided

Only common tests should be described solely by name; describe more complex techniques in the Methods section.

$\bigotimes$ A description of all covariates tested

Х A description of any assumptions or corrections, such as tests of normality and adjustment for multiple comparisons

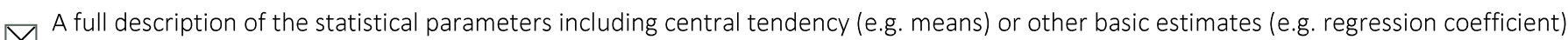

AND variation (e.g. standard deviation) or associated estimates of uncertainty (e.g. confidence intervals)

For null hypothesis testing, the test statistic (e.g. $F, t, r$ ) with confidence intervals, effect sizes, degrees of freedom and $P$ value noted Give $P$ values as exact values whenever suitable.

Х $\square$ For Bayesian analysis, information on the choice of priors and Markov chain Monte Carlo settings

Х $\square$ For hierarchical and complex designs, identification of the appropriate level for tests and full reporting of outcomes

$\triangle \mid \square$ Estimates of effect sizes (e.g. Cohen's d, Pearson's $r$ ), indicating how they were calculated

\section{Our web collection on statistics for biologists contains articles on many of the points above.}

\section{Software and code}

Policy information about availability of computer code

Data collection SQL Server Management Studio v18.5.1, Seegene proprietary software.

Data analysis Python 3.7.1. Including: scipy (v1.1.0) and statsmodels (v0.9.0).

For manuscripts utilizing custom algorithms or software that are central to the research but not yet described in published literature, software must be made available to editors and

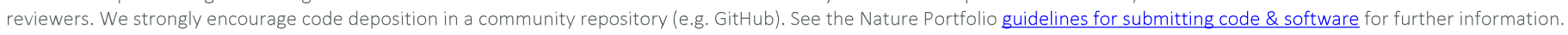

\section{Data}

Policy information about availability of data

All manuscripts must include a data availability statement. This statement should provide the following information, where applicable:

- Accession codes, unique identifiers, or web links for publicly available datasets

- A description of any restrictions on data availability

- For clinical datasets or third party data, please ensure that the statement adheres to our policy

According to the Israel Ministry of Health regulations, individual-level data cannot be shared openly. Specific requests for remote access to de-identified data should be referred to KSM, Maccabi Healthcare Services Research and Innovation Center. Requests sent to Sarit Chehanowitz (chehanow_s@mac.org.il) will be considered within 21 days pending IRB approval and MHS regulations. 
Please select the one below that is the best fit for your research. If you are not sure, read the appropriate sections before making your selection.

\ Life sciences

Behavioural \& social sciences

Ecological, evolutionary \& environmental sciences

For a reference copy of the document with all sections, see nature.com/documents/nr-reporting-summary-flat.pdf

\section{Life sciences study design}

All studies must disclose on these points even when the disclosure is negative.

Sample size Sample size was not restricted. It was only limited by the number of patients with breakthrough infections.

Data exclusions We have excluded patients under 20 years old, as this group wasn't approved yet for booster shot, and was lately approved for the vaccine at all. This exclusion criterion was pre-established.

Replication We report the results of an observational study.

Randomization Not randomized. In our analysis we used linear regression model, making randomization unneeded.

Blinding We report the results of an observational study.

\section{Reporting for specific materials, systems and methods}

We require information from authors about some types of materials, experimental systems and methods used in many studies. Here, indicate whether each material, system or method listed is relevant to your study. If you are not sure if a list item applies to your research, read the appropriate section before selecting a response.

Materials \& experimental systems

$\mathrm{n} / \mathrm{a}$ Involved in the study

Х $\square$ Antibodies

Methods

\ $\square$ Eukaryotic cell lines

$\mathrm{n} / \mathrm{a}$ Involved in the study

Х $\square$ Palaeontology and archaeology

X $\square$ ChIP-seq

Х $\square$ Animals and other organisms

Х $\square$ Flow cytometry

$\square \bigotimes$ Human research participants

\ $\square$ Clinical data

$\bigotimes \mid \square$ Dual use research of concern

\section{Human research participants}

\section{Policy information about studies involving human research participants}

Population characteristics

Recruitment

Ethics oversight
Patients above 20 years old infected with SARS-CoV-2: 3,100 unvaccinated (1,304 males and 1,796 females); 12,934 2-dose vaccinated (5,785 males and 7,149 females); 519 booster vaccinated (290 males and 229 females).

\section{This is an observational study involving electronic health records analysis}

This study was approved by the MHS (Maccabi Healthcare Services) Institutional Review Board (IRB) 